\title{
METACARPOPHALANGEAL PATTERN PROFILE ANALYSIS IN 14 JAPANESE CHILDREN WITH SOTOS SYNDROME
}

\author{
Yasushi Takahashi, * Kiyoshi Imaizumi, Fumio TaKada, ** \\ and Yoshikazu Kurokı \\ Division of Medical Genetics, Kanagawa Children's Medical Center, \\ Yokohama, Kanagawa 232, Japan
}

\begin{abstract}
Summary Metacarpophalangeal pattern profile (MCPP) was analyzed in 14 Japanese children (mean age 6.7 years old) with Sotos syndrome. The patients were divided into 2 groups based on age; group $1 \quad(n=8)$ : 6 years or over; group $2(n=6)$ : less than 6 years. The mean values of standard deviation of the 14 patients with obviously large hand were all above 1.4. The MCPP in group 1 showed (1) two major peaks in metacarpal and proximal phalangeal areas, (2) a small peak in middle phalangeal area, and (3) relatively short distal phalangeal bones compared with the metacarpal and proximal phalangeal bones. The MCPP in group 2 was similar to that in group 1, but an additional peak was observed in distal phalangeal area. The MCPP of Japanese patients showed a quite similar pattern to that of Caucasian patients, and we conclude the method can also be a useful tool in the diagnosis of the Japanese patients. In correlation study, 8 of the 14 patients had a significant positive correlation, but 2 patients in group 2, less than 3 years, had no positive correlation. We suggest the method is not applicable to young patients less than 3 years.

Key Words metacarpophalangeal pattern profile (MCPP), Sotos syndrome
\end{abstract}

\section{INTRODUCTION}

Sotos syndrome, first described by Sotos et al. (1964), is characterized by large size at birth, large hands and feet, advanced osseous maturation, macrocephaly

Received November 5, 1993; Revised version accepted January 7, 1994.

Present address: *Department of Pediatrics, Akita University School of Medicine, Akita, Japan.

**Department of Pediatrics, Kitasato University School of Medicine, Yokohama, Japan. 
with prominent forehead, down-slanting palpebral fissures, hypertelorism, prognathism, abnormal coordination, and variable degrees of mental retardation. Other occasional findings of the syndrome include urogenital anomalies (Moriyama et al., 1984), congenital heart defects (Kaneko et al., 1987), and malignancies (Dodge et al., 1983; Hersh et al., 1992).

The diagnosis of Sotos syndrome depends on a constellation of clinical manifestations (Wit et al., 1985). Metacarpophalangeal pattern profile (MCPP) analysis involves an evaluation of the hand skeleton based on a comparison of 19 tubular bone lengths to normal bone lengths standards, as described by Garn et al. (1972) and Poznanski et al. (1972). It thus would help the diagnosis of Sotos syndrome (Butler et al., 1985, 1988). Present study deals with MCPP analysis in 14 Japanese children with the syndrome, and evaluates whether the method is applicable to young patients.

\section{MATERIALS AND METHODS}

A total of 14 children ( 8 boys and 6 girls) with Sotos syndrome were enrolled in the study. All of them were individually admitted to the Division of Medical Genetics, Kanagawa Children's Medical Center during 1979 to 1993 . The diagnosis of Sotos syndrome was confirmed independently by two clinical genetists. Their age ranged from 1.9 to 11.2 years with a mean of 6.7 years.

Informed consent concerning the present study was obtained from the parents of each child. Postero-anterior hand radiographs were taken, and the lengths of the 19 matacarpophalangeal bones on the right hand were measured in $10^{-1}$ millimeter by a Vernier caliper. The values of standard deviation for the 19 bones were calculated by comparing them to standard bone lengths of Japanese children (Matsuura and Kajii, 1989), and the individual pattern profiles were obtained. The patients were divided into 2 groups based on age; group $1(n=8): 6$ years or over; group $2(n=6)$ : less than 6 years, and the mean pattern profiles (MCPP) in each group were obtained from the individual profiles. All measurements were carried out by one of the authors (Y.T.). The measurements and calculations of MCPP analysis were performed with the method described by Garn et al. (1972) and Poznanski et al. (1972). Correlation studies were performed to assess similarity between the individual patterns and the mean pattern profile, and a p-value of 0.05 or less was considered statistically significant.

\section{RESULTS}

The mean pattern profile in both group 1 and 2 were shown in Fig. 1. The mean values of standard deviation in group 1 were all above 1.5. The mean pattern profile in group 1 showed two major peaks in the metacarpal and proximal phalangeal areas and a small peak in the middle phalangeal area. The distal phalangeal 


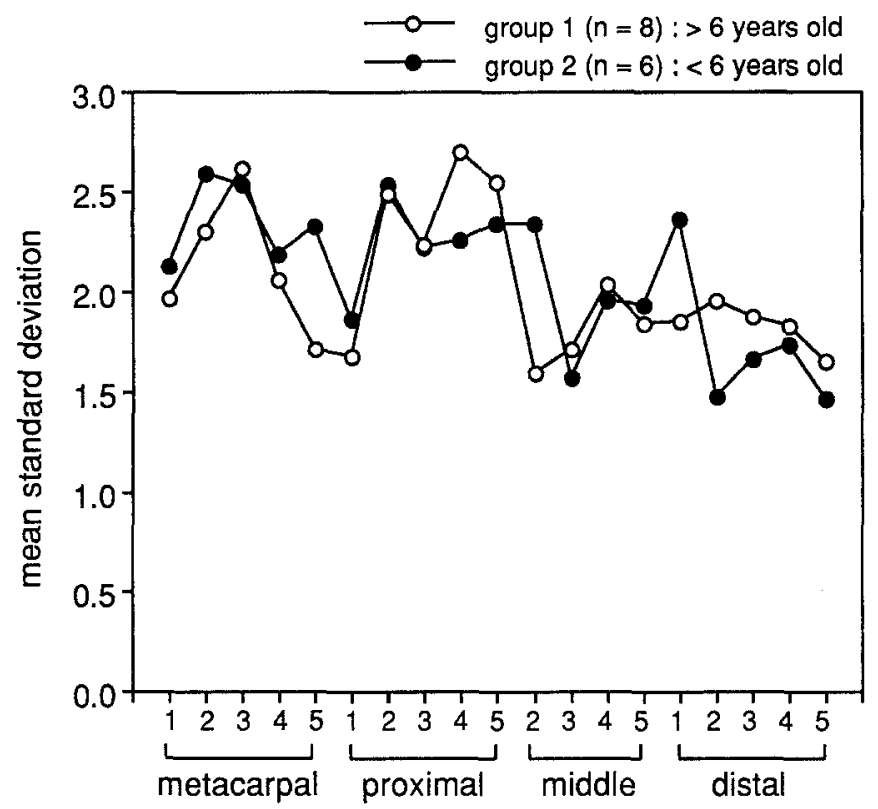

Fig. 1. Mean MCPP in group 1 (open circle) and group 2 (closed circle).

Table 1. Correlation between Sotos syndrome individual's MCPP and group mean MCPP.

\begin{tabular}{cccc}
\hline Group & Age (yrs) & Sex & Correlation \\
\hline Group 1 $>6.0$ yrs) & 11.2 & M & 0.40 \\
& 10.3 & M & $0.57^{*}$ \\
& 10.0 & M & $0.52^{*}$ \\
& 9.7 & F & $0.69^{*}$ \\
& 9.3 & F & $0.72^{*}$ \\
& 8.9 & M & 0.41 \\
Group 2 (<6.0 yrs) & 8.2 & F & 0.36 \\
& 7.1 & M & 0.30 \\
& 5.9 & F & $0.78^{*}$ \\
& 5.8 & F & $0.85^{*}$ \\
& 5.2 & M & $0.78^{*}$ \\
& 3.0 & M & $0.59^{*}$ \\
& 2.7 & F & -0.15 \\
& 2.5 & M & -0.13 \\
\hline
\end{tabular}

yrs, years; $M$, male; F, female; ${ }^{*} \mathrm{p}<0.05$.

bones were relatively short compared with the metacarpal and proximal phalangeal bones. The mean values of standard deviation in group 2 were all above 1.4. The mean pattern profile in group 2 showed a pattern similar to that in group 1 , but a major peak was observed in the distal phalangeal area in addition to the two major peaks in metacarpal and proximal phalangeal areas. 
The correlation of each individual is shown in Table 1. Four of the 8 patients in group 1 and 4 of the 6 patients in group 2 had a significant positive correlation. Remaining 4 patients in group 1 had a weak positive correlation, but 2 patients in group 2, less than 3 years old, had no positive correlation.

\section{DISCUSSION}

MCPP analysis on Sotos syndrome was performed by Dijkstra et al. (1985) and Butler et al. (1985). Butler et al. (1988), in a follow-up of their 34 subjects, stated that (1) the mean values of standard deviations in the patients with Sotos syndrome fall between 1.8 to 3.7 , (2) the MCPP profile contains one major peak in the proximal phalangeal area and a smaller peak in the metacarpal area, and (3) the distal bones are relatively short compared with the proximal bones. The MCPP profile in the present series of Japanese patients showed a quite similar pattern to that in Caucasian patients. Thus, we suggest that there is no racial difference between the Japanese and Caucasians in the MCPP in Sotos syndrome, and that the method is useful in the diagnosis of the Japanese patients with the syndrome.

There was no apparent difference in the MCPP between the 6 younger patients and the older 8 patients, indicating that the method is applicable to young patients. However, we have to notice a limitation of the age level in the method. The epiphysis is included in the measurement of the hand tubular bones in MCPP analysis, but, it does not always appear at the ages of less than 3 years. In correlation study, no positive correlation was observed in the youngest 2 patients, both less than 3 years. Thus, the method can not be applicable to young patients less than 3 years.

In conclusion, the MCPP analysis is useful in the diagnosis of Sotos syndrome in Japanese, and this method is applicable for Sotos syndrome patients at age 3 years or over.

Acknowledgnents The authors wish to thank Dr. Shinya Matsuura and Dr. Tadashi Kajii for excellent advice.

\section{REFERENCES}

Butler MG, Meaney FJ, Kittur S, Hersh JH, Hornstein L. (1985): Metacarpophalangeal pattern profile analysis in Sotos syndrome. Am J Med Genet 20: 625-629

Butler MG, Dijkstra PF, Meaney FJ, Gale DD (1988): Metacarpophalangeal pattern profile analysis in Sotos syndrome: a follow-up ieport on 34 subjects. Am J Med Genet 29: 143-147

Dijkstra PF (1985): Cerebral gigantism (Soto's syndrome). Metacarpophalangeal pattern profiles. Fortschr Röntgenstr 143: 183-185

Dodge PR, Holmes SJ, Sotos JF (1983): Cerebral gigantism. Dev Med Child Neurol 25: 248-252

Garn SM, Hertzog KP, Poznanski AK, Nagy JM (1972): Metacarpophalangeal length in the evaluation of skeletal malformation. Radiology 105: 375-381 
Hersh JH, Cole TRP, Bloom AS, Bertolone SJ, Hughes HE (1992): Risk of malignancy of Soto's syndrome. J Pediatr 120: 572-574

Kaneko H, Tsukahara M, Tachibana H, Kurashige H, Kuwano A, Kajii T (1987): Congenital heart defects in Sotos sequence. Am J Med Genet 26: 569-576

Matsuura S, Kajii T (1989): Radiographic measurements of metacarpophalangeal lengths in Japanese children. Jpn J Human Genet 34: 159-168

Moriyama M, Terashima K, Fukushima Y, Kuroki Y (1984): Urogenital anomalies in patients with Sotos syndrome. Jpn J Urol 75: 591-593 (in Japanese with English abstract)

Poznanski AK, Garn SM, Nagy JM, Gall JC (1972): Metacarpophalangeal pattern profiles in the evaluation of skeletal malformations. Radiology 104: 1-11

Sotos JF, Dodge PR, Muirhead D, Crawford JD, Talbot NB (1964): Cerebral gigantism in childhood: A syndrome of excessively rapid growth with acromegalic features and a non progressive neurological disorder. N Engl J Med 271: 109-116

Wit JM, Beemer FA, Barth PG, Oorthuys JWE, Dijkstra PF, Van den Brande JL, Leschot NJ (1985): Cerebral gigantism (Sotos syndrome). Compiled data of 22 cases. Eur J Pediatr 144: $131-140$

Vol. 39, No. 1, 1994 